\title{
Research on Public Space Renewal Strategy of Small and Medium-sized Towns Based on Spatial Syntax--Taking Liulin Liaoyuan Square as an Example
}

\author{
Zhou Yingzheng ${ }^{1}, \mathrm{Xu}$ Qiang $^{2}$ \\ ${ }^{1}$ Taiyuan University of Technology,graduate student \\ ${ }^{2}$ Taiyuan University of Technology, associate professor
}

\begin{abstract}
With the accelerating urbanization, the living standards of people in small towns are constantly improving. There are more or less problems in the public spaces that were used in the past, whether planned or spontaneously formed. Today, under government-led conditions, we have the ability to improve the spatial quality and life satisfaction of these public spaces. This paper takes Liulin Liaoyuan Square as an example, mainly for the transformation and renewal of such small and medium-sized public spaces. It compares the current situation with the simulation implementation plan, uses the spatial syntax including direct (non-participant) observation and spatial grammar analysis techniques to update the strategy and provide practical advice on practical problems.
\end{abstract}

\section{Introduction}

A city center with great attraction plays an important role in the city's livability scoring system. Improving the comfort of our residents also requires an attractive public living space. The town is dynamic, and the dynamic relationship with non-stop birth, progress, development will always follow our time to develop. The central square of town is a key and must continue to develop.

In recent years, the use of spatial syntax in urban planning and other fields has achieved a series of research results. For example, Hu Haili applies in the transportation space planning of Zhangjiang New District in Zhangzhou; Wang Chengfang and Guo Xiangyu applies in the historical district protection and renewal planning of road traffic and spatial relations; Zhou Wei uses Axwoman space syntax software in the gis platform to analyze the historical district of Confucius Temple in Nanjing and puts forward reasonable suggestions in combination with commercial format; and foreign scholar Rim Meziani uses space syntax to locate Abu Dhabi public transportation stations (bus, subway and tram) and other schools and does research on walking accessibility between public settings. In general, the spatial syntax is widely used, but there are few examples of using spatial syntax to solve practical problems in public spaces such as squares.

This paper takes the public space of the entrance plaza of Mingqing Street in Liulin County as an example. On the basis of the former researchers, the space public syntax is used to transform the public space of the square into a scene where the space syntax can be applied. It can use CAD to provide some ideas and solutions for the design of public spaces.

\section{Overview of research areas and research methods}

\subsection{Research area}

Liulin Street is located in the center of Liulin County and is the original Liulin Ancient Town. It is an important road for merchants in history. With hundreds of years' historical and cultural accumulation, $g$ Street has gradually formed a block with rich commercial culture and life. The public space of the former square at the entrance of Mingqing Street and the Liulin Hotel is the key space for this study. Liaoyuan is named after the Liaoyuan Building on the west side of the square. It is located at the intersection of Mingqing East Street, National Highway 307 and Hechang Street. It is the entrance public space on the east side Mingqing Street, with most of the vehicles entering and leaving Liulin. This makes Liaoyuan Square a famous place for Liulin County. The former square of Liulin Hotel was originally the inner space of the hotel. After the government negotiated, the entire area will be re-planned and the space in front of the hotel will be opened.

With the continuous development of Liulin's economy and society, the problems of Mingqing Street and the entrance of the square are constantly exposed. Not only are the building facilities and circuit facilities old, the traffic roads blocked, the squares parked in a jumble, etc., but also the design of public spaces is inelastic and not targeted. The comfort does not meet the needs of the crowd. In the process of upgrading and 
rebuilding, new problems are constantly being generated, such as the relationship between the Liaoyuan Square and the former square of Liulin Hotel brought about by the re-planning of urban roads in the region, and the relationship between these public spaces and the cultural relics protection group called Xiangyan Temple. And the problem of the integration of traditional business formats and modern business formats after the renovation and renewal is also existed. (Figure 1)

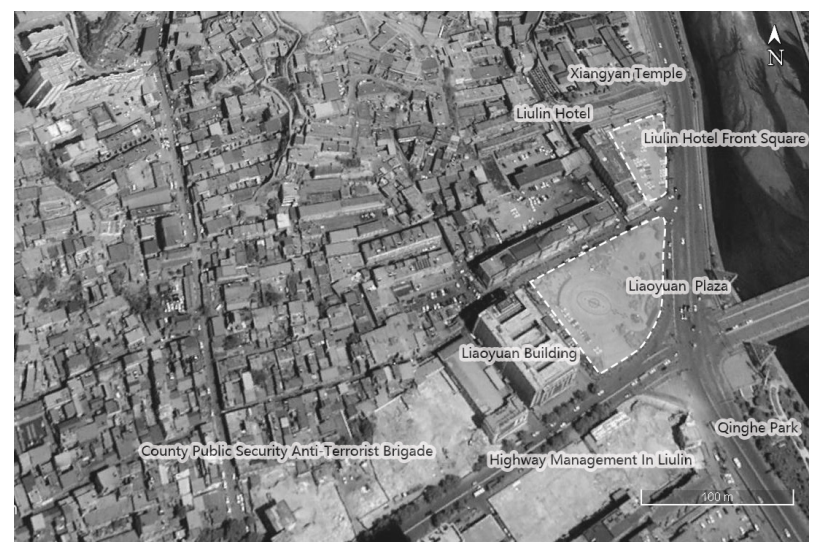

Fig. 1. The status quo of Liulin

\subsection{Research methods}

As digital technology revolutionizes the way urban planning is produced, the risk of planning without theory to become a practice is growing. By describing the systematic relationship between usage patterns and spatial phenomena, spatial grammar helps us to study urban planning. Spatial syntax is a part of the application of topological analysis in urban planning. The network organization of urban streets is understood as a connection method that gets less connections through any street in the network. The advantage of this approach is that it is supported by a powerful theory of space society.

Axis analysis is the core method of spatial syntax method, which is used to study the influence of street patterns on related sports and motion functions. Among them, the degree of integration is the collection of those streets with the highest accessibility, and the general improvement of accessibility can bring about changes in land use. Because of the high accessibility, the commercial and cultural values surrounding these roads have potentials for development. The article analyzes the current situation around the square and the simulation plan separately, and compares the changes of the values before and after the planning to guide the planning to complete better.

In particular, in order to facilitate the analysis of the Liaoyuan Square, the Mingqing East Street continues to extend eastward, and the exit position of the square has also been extended to the south and north. This can be seen as a Axis analysis of the flow of people, and control the road range, in order to obtain more objective experimental results.

\section{Analysis of using axis analysis}

\subsection{Connection value result analysis}

The connection value represents the number of spaces in a space in the system. The higher the connection value is, the better the spatial permeability is. From the results, it can be concluded that the current road connection value around the Liaoyuan Square is 4, 10, 3, and 4 in the east, south, west, and north (Figure 2). The road connection values around the Liaoyuan Square are planned to be 6 , 9,3 , and 9 in the east, south, west, and north respectively, and the connection value of the street in Mingqing Street is 13 (Figure 3). From a commercial point of view, whether the road is accessible or not is a very important factor, and the value of the road connection with this space is also very important. Generally speaking, the road connection value in the town center must be relatively high. Therefore, the level of the connection value reflects to some extent whether the regional road can become the center of the town.

Comparing the current situation with the planned road network, it can be seen that the planned road has obviously strengthened the connection value of Mingqing Street, which has increased the connection value of the roads around the Liaoyuan Square, indicating the increase of the roads around Mingqing Street and Liaoyuan Square. The road network is more dense, and the space penetration in Liulin County is better. The Luanyuan Square will also be one of the most important squares in Liulin County.

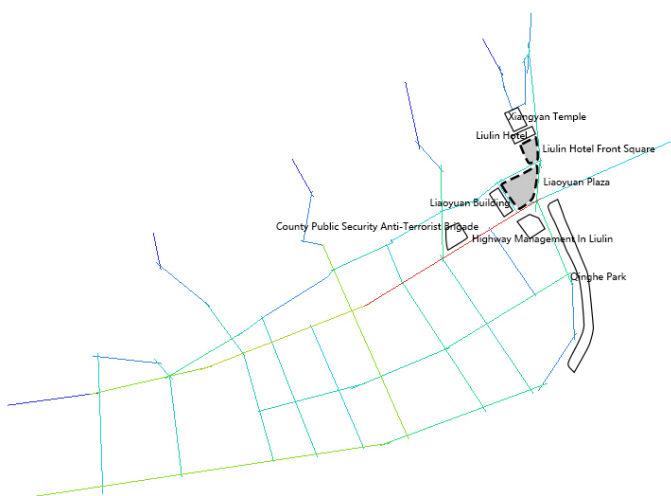

Fig. 2. Current Connection Value

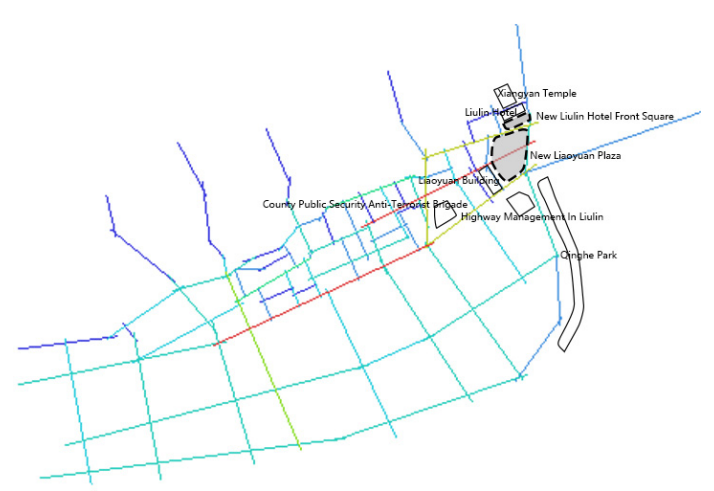

Fig. 3. Planning connection value 


\subsection{Reachability analysis}

The space with high integration value has high accessibility, which can measure the potential of space to attract traffic. The road integration value (Fig. 4) in the east of the area is 1.60425; the south road integration value is 2.65915; the west road integration value is 1.79149 ; the north road integration value is 1.604258 . After the planning, the integration value of the road in the east of the Liaoyuan Square (Fig. 5) is 2.37946; the road integration value in the south is 2.66579; the road integration value in the west is 2.0399 by the Liaoyuan Building; the road integration value is 2.018068 by the passenger station; the road integration value in the north is 2.40416; and the integration value of Mingqing Street is 2.77992 (Table 1).

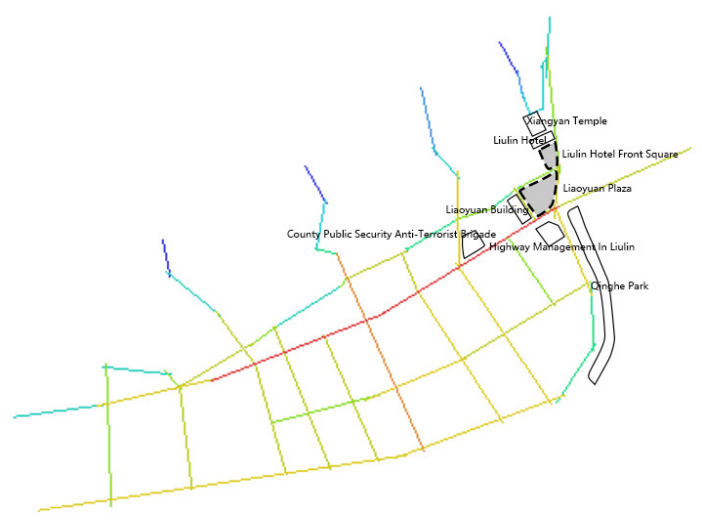

Fig. 4. Current integration value

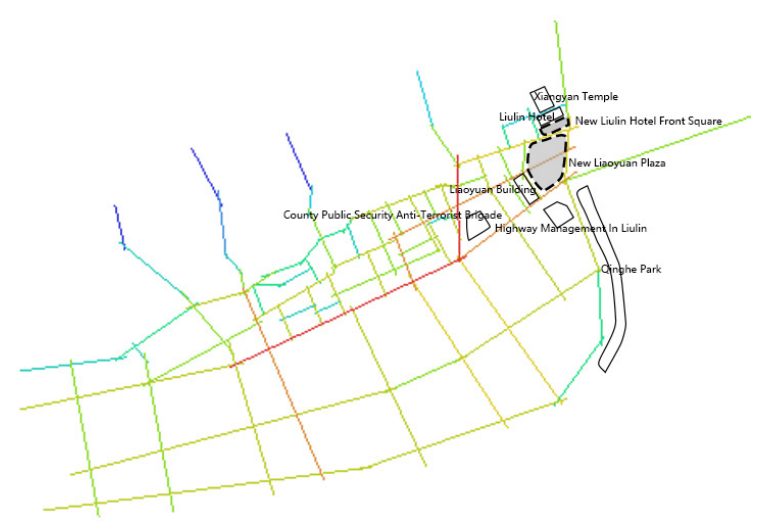

Fig. 5. Planning integration value

Comparing the two figures, it can be seen that the color of the roads around the original Liaoyuan Square is lighter than before. Therefore, the road integration value of the surrounding area of the Liaoyuan Square is lower than that in Liulin County after the planning, but the value is different from the previous one. The ratio is higher, which means that the accessibility of the county town of Liulin County has been improved, and the accessibility of the entire Mingqing Street has been improved.
Table 1. Comparison of two scheme integration values

\begin{tabular}{c|c|c|c|c|c}
\hline Planning & East & South & \multicolumn{2}{|c|}{ West } & North \\
\hline $\begin{array}{c}\text { Before } \\
\text { planning }\end{array}$ & 1.60425 & 2.65915 & \multicolumn{2}{|c}{1.79149} & 1.604258 \\
\hline $\begin{array}{c}\text { After } \\
\text { planning }\end{array}$ & 2.37946 & 2.66579 & 2.0399 & 2.018068 & 2.40416 \\
\hline $\begin{array}{c}\text { Mingqing } \\
\text { Street }\end{array}$ & & & & \\
\hline
\end{tabular}

\subsection{Intelligence results analysis}

In addition, the comparison of intelligence data, that is, the correlation coefficient (intelligence) between global integration and local integration is the mathematical fitting of local and global correlation. The higher the correlation coefficient is, the better the surface space coordination is. This paper is limited to three topological distances to express the reachability of walking.

It is obtained that the current fitness $\mathrm{R} 2$ is 0.705305 (Fig. 6), and the fittness $\mathrm{R} 2$ after the planning is 0.862899 (Fig. 7). The larger mark in the figure indicates the intelligence of the road around the square, and the planned road mark is closer to the fitted line in the middle.

It can be seen from the data that the correlation coefficient between global integration degree and local integration degree after planning is higher than that before planning. Therefore, the correlation between urban global and local space after planning is stronger than the current situation. Judging from the roads around the Liaoyuan Square selected in the map, their position after the planning is better than that before the planning, so it is considered that the relationship between the original Liaoyuan Square and the entire Liulin County will be strengthened after the planning, which is conducive to the self-improvement optimization of urban space.

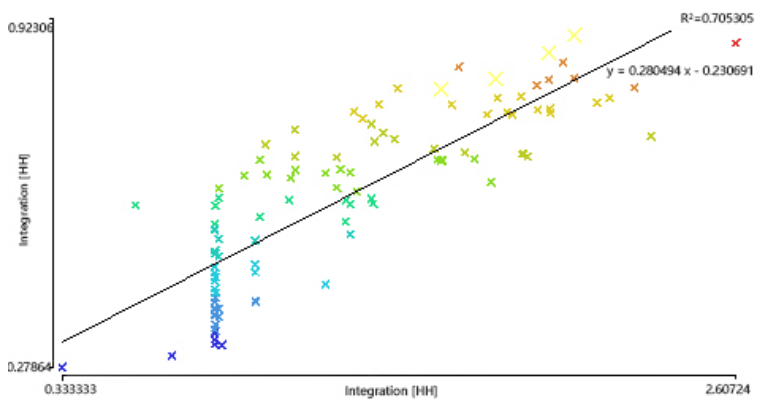

Fig. 6. Current fittness R2 


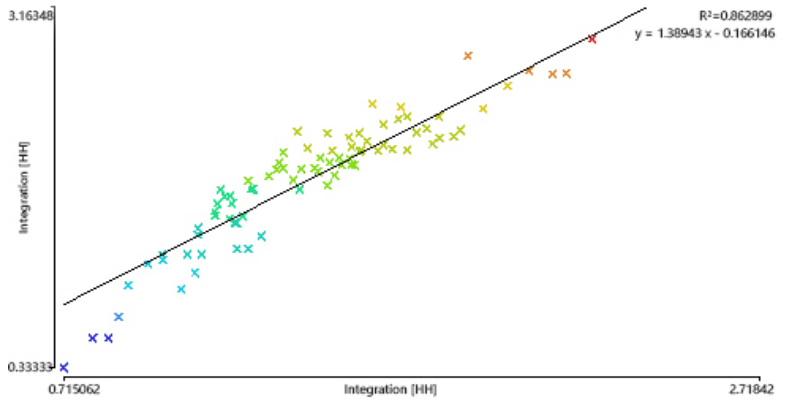

Fig. 7. Planning fittnessR2

\section{Analysis of actual problems}

\subsection{Relationship between the original Liaoyuan Square and Liulin Hotel after planning}

Xiangyan Temple is a cultural relics protective building in Liulin County. As a temple, its incense is very strong. Located in the north of Liulin Hotel, the front square of Liulin Hotel may use the same guiding space with Xiangyan Temple. Therefore, in terms of facilitating the local pilgrimage group, or attracting foreign tourists to visit, it is necessary to upgrade the road around Xiangyan Temple, which is the goal of planning the accessibility of the former square of Liulin Hotel. From the experimental results, the integration value of the roads around the former square of Liulin Hotel after planning is $1.79333,2.40416,1.61786,1.14606$ in the East, South, West and North. There is still a certain gap compared with the higher integration values of Mingqing Street (2.77792) and Liaoyuan Square (East, South, West, North 2.37946, 2.66579, 2.0399, 2.40416) (Table 2), indicating that few people scattered from the Liaoyuan Square will arrive at the planned former Liulin Square and visit the Xiangyan Temple.

Table 2. Comparison of the road around the front square of Liulin Hotel and the Liaoyuan Square after the planning integration values

\begin{tabular}{c|c|c|c|c|c}
\hline Planning & East & South & \multicolumn{2}{|c|}{ West } & North \\
\hline $\begin{array}{c}\text { The road around } \\
\text { the front square } \\
\text { of Liulin Hotel }\end{array}$ & 1.79633 & 2.40416 & \multicolumn{2}{|c}{1.61786} & 1.14606 \\
\hline $\begin{array}{c}\text { The Liaoyuan } \\
\text { Square after the } \\
\text { planning }\end{array}$ & 2.37946 & 2.66579 & 2.0399 & 2.018068 & 2.40416 \\
\hline Mingqing Street & & & & & \\
\hline
\end{tabular}

Based on this data, it is recommended to improve the relationship between the planned Liaoyuan Square and former square of Liulin by setting obvious pedestrian crossings, erecting pedestrian bridges or underground passages to improve the accessibility of the road south of former square of Liulin and attracting people to Xiangyan Temple.

\subsection{The bus station settings after planning}

The original bus station is located on the sides of the National Highway 307 in the east of Liulin Hotel. The obvious problem is that the station space is relatively narrow, about two meters wide, and it occupies the crosswalk. As a national road, there are more vehicles here and there the safety of people who are waiting for the car is not guaranteed. After the road planning, the national road bypasses the outer side of the town; the car in front of the bus stop is reduced; and the safety problem is relieved. However, the location of the bus station after planning is still worth discussing. Considering several aspects of bus station location, such as passenger convenience, passenger activity safety, traffic traversal at road intersections, and convenience of passenger transfer, it is necessary to make it convenient for people to get to the desired location from the station. It is necessary to set the station near the road with high accessibility and set it downstream of the road intersection to reduce the impact on the normal driving vehicle as far as possible, make it easy for people to find the location of the new station at the same time, and try not to stay away from the current station location.

According to the accessibility analysis, the accessibility of the East Street and its extension line in Mingqing Street is relatively high. It is at the downstream of the road intersection, and the distance from the original station is suitable. Therefore, it is recommended to set up the new station in the MingQing East Street and the intersection of the line of the original national road.

\section{Summary}

The success of the design of public places in town squares is not only to see whether the connection value, accessibility, intelligence, etc. have met the requirements, but also whether the most common needs of people have been met. The success or failure of a space is likely to be on some seemingly small factors.

When paying attention to spatial renewal, we should pay more attention to the individual needs of people. When the individual needs of the residents living around are satisfied, the revival of the town center square can be more timely, making people be more willing to approach the town center square. On the basis of previous studies, the article attempts to transform spatial syntax into a means of solving the public space through the combination of practice and theory. Although it is not perfect, it hopes to provide a research idea.

\section{References}

1. Hu Haili, Ren Yilin, Huang Yingmin, Chen Jinquan. Study on Planning Traffic Space Evaluation of Zhangjiang New Area in Zhangzhou Based on Spatial Syntax and GIS[J].Journal of Jiangxi University of Science and Technology,2017,38(05):13-18. 
2. Wang Chengfang, SunYimin. Study on the Method of Historical District Protection and Renewal Planning Based on GIS and Space Syntax-

Taking Jiangmen Historical District as an Example[J].Tropical Geography,2012,32(02):154159.

3. Guo Xiangmin,Wang Jincan. Study on the Renewal Strategy of Public Space in Dongmen Old Street in Shenzhen Based on Spatial Syntax[J].Planner,2014,30(05):89-95.

4. Zhou Wei. Study on the Distribution Characteristics and Mechanism of Spatial Pattern of Tourism Historic District-Taking Nanjing Confucius Temple as an Example[J].Commercial Research,2016(05):179-186.

5. Meziani. Accessibility and Integration Study of Part of the Abu Dhabi 2030 Master Plan by Using Space Syntax [J]. Civil Engineering and Architecture: English Edition, 2017(1): 81-90.

6. Wang Wei. Empirical Study of Urban Form Based on GIS and Spatial Syntax[J]. Geographic Information World, 2016, 23(03): 119-122.

7. MA Wei, CHENG Wei, XU Xiao-yan. Commercialization Renewal and Public Space Optimization Model of Urban Village Based on Spatial Syntax[J]. Engineering and Construction, 2014, 28(04): 468-470+552. 